\title{
Title: \\ The Detectability of Asteroids and Comets Before Earth Impact
}

Author(s):

Jack Hills, T-6

Submitted to:

DOE Office of Scientific and Technical Information (OSTI)
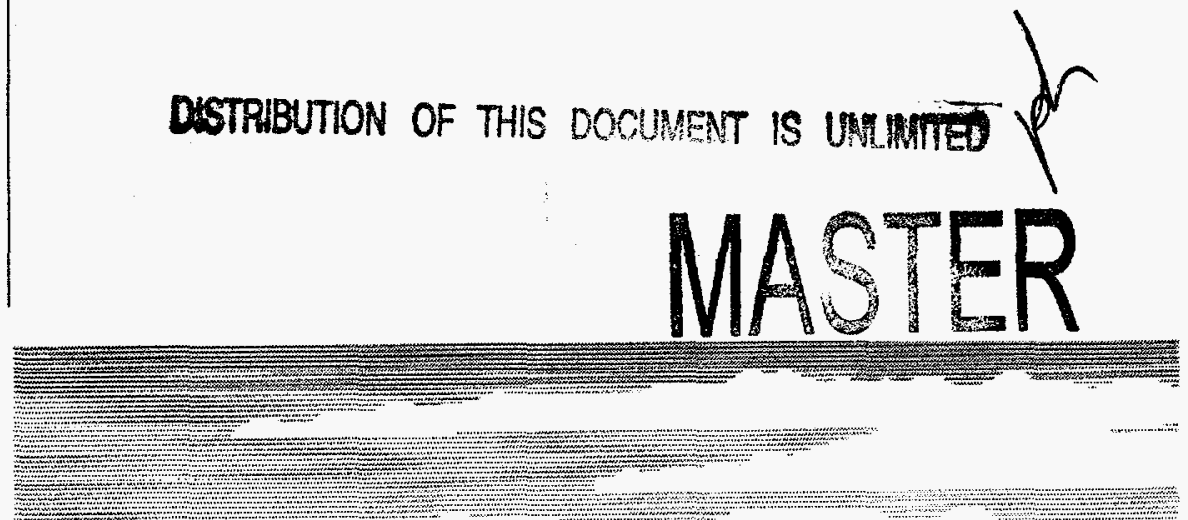

\section{Los Alamos}

NATIONAL LABORATORY

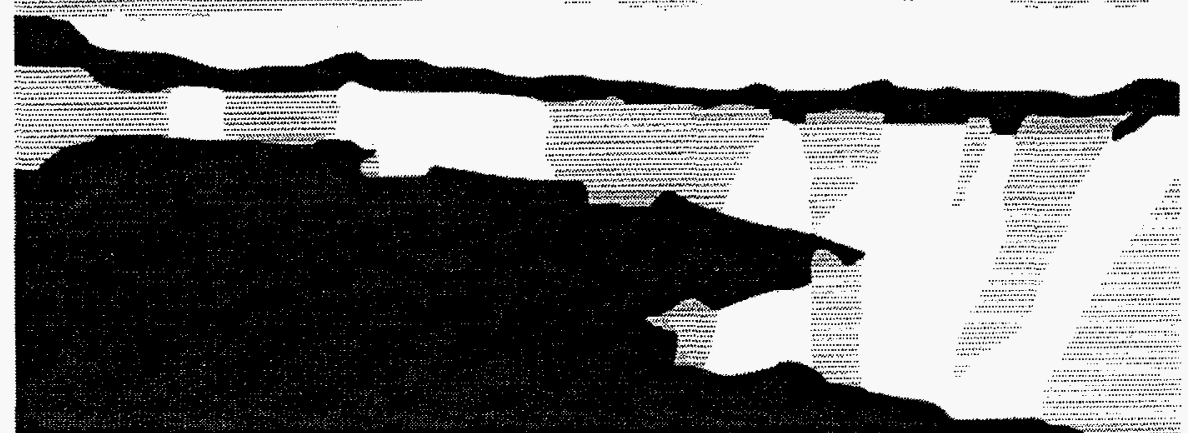

Los Alamos National Laboratory, an affirmative action/equal opportunity employer, is operated by the University of California for the U.S. Department of Energy under contract W-7405-ENG-36. By acceptance of this article, the publisher recognizes that the U.S. Government retains a nonexclusive, royaltyfree license to publish or reproduce the published form of this contribution, or to allow others to do so, Ior U.S. Government purposes. The Los Alamos National Laboratory requests that the publisher identify this article as work performed under the auspices of the U.S. Department of Energy. 
DISCLAIMER

Portions of this document may be illegible in electronic image products. Images are produced from the best available original document. 


\section{DISCLAIMER}

This report was prepared as an account of work sponsored by an agency of the United States Government. Neither the United States Government nor any agency thereof, nor any of their employees, makes any warranty, express or implied, or assumes any legal liability or responsibility for the accuracy, completeness, or usefulness of any information, apparatus, product, or process disclosed, or represents that its use would not infringe privately owned rights. Reference herein to any specific commercial product, process, or service by trade name, trademark, manufacturer, or otherwise does not necessarily constitute or imply its endorsement, recommendation, or favoring by the United States Government or any agency thereof. The views and opinions of authors expressed herein do not necessarily state or reflect those of the United States Government or any agency thereof. 


\title{
The Detectability of Asteroids and Comets \\ Before Earth Impact
}

\author{
Jack Hills*
}

\begin{abstract}
This is the final report of a one-year, Laboratory-Directed Research and Development (LDRD) project at the Los Alamos National Laboratory (LANL). We investigated the requirements for detecting asteroids and comets in the final years before they impact Earth and determined the equipment and strategies needed for detecting impacting asteroids and comets in all their permitted orbits. When this information is combined with the properties of possible mechanisms for diverting asteroids, it will determine the degree of readiness and the minimum capability of the deflection mechanisms required to prevent impact of these objects with Earth.
\end{abstract}

\section{Background and Research Objectives}

It has been increasingly evident that comet and asteroid impacts have been a major source of stress on the biosphere of Earth. A 10-km object destroyed the dinosaurs and more than half the life on Earth. Comet Hale-Bopp, the comet of the century, which is scheduled to cross the orbit of Earth in 1997 maybe $100 \mathrm{~km}$ in diameter and could sterilize Earth if it were to hit (which it will not this trip). Smaller objects hit more frequently, but would still cause unacceptable regional and global damage.

It has been realized in recent years that this damage is preventable with present technology. NASA, DOE, and the U.S. Air Force have in recent years investigated aspects of the problem and all these entities will probably play some role in the eventual solution. One of the current bottlenecks is detection strategies. Academic investigators have proposed to NASA a battery of six 3-meter, wide-field telescopes (Spaceguard) that would cost over $\$ 200$ million dollars to build and over $\$ 10$ million dollars a year to operate. They supposedly would detect about $95 \%$ of the Earth-crossing asteroids over the

\footnotetext{
*Principal investigator, e-mail: jgh@lanl.gov
} 
next 25 years, but we believe that the search strategies suggested for these telescopes would only allow about $80 \%$ of the potential impactors to be detected. However, a change in search strategy would allow over $90 \%$ of any objects that will impact Earth over the next 100 years to be detected with much smaller telescopes, such as the existing 1-meter GEODDS telescopes operated by Space Command. The Air Force appears interested in supporting such a program, if it is effective. A major detection program, which is the most critical element on the critical path for solution of the problem, would allow other parts of the project to proceed. The objective of this LDRD project was to investigate changes in strategy that would make the detection faster and cheaper.

\section{Importance to LANL's Science and Technology Base and National R\&D Needs}

Previous work in this area at Los Alamos has principally been in identifying the amount and types of damage that these impacting objects can cause and in finding methods to deflect them before Earth impact. The detection problem is closely tied to the deflection problem. Previous work at LANL has found the minimum time required to deflect objects as a function of their size. Using this information, we can devise strategies to detect Earth-impacting objects in sufficient time to prevent impact. The solution of the detection problem is a necessary first step towards addressing this potentially devastating threat to life on Earth.

\section{Scientific Approach and Results}

We examined strategies for detecting Earth-Crossing Asteroids (ECAs) that may hit Earth within the next few years. We wish to detect asteroids down to 60 meters in diameter in sufficient time to allow them to be deflected or destroyed before Earth impact. Work at LANL has shown that a week is sufficient warning to allow a single rocket equipped with a nuclear explosive (using existing rocket booster sand nuclear explosives) to deflect from Earth impact an asteroid with a diameter up to $1-2 \mathrm{~km}$, if such a rocket were on standby for this purpose. To deflect a larger asteroid requires a lead time of months to years even if rockets to deflect it were on standby.

These lead times suggest that objects less than about $1 \mathrm{~km}$ in diameter need only be detected a week or two before impact as part of a terminal defense system. In the work supported by this project, we have found that telescopes as small as 5 inches in diameter would be sufficient to detect objects larger than 100 meters in diameter at 10 days to impact if they approach Earth in the hemisphere facing away from the sun. (The object would look full in the part of the sky opposite the sun and would look increasingly like a faint crescent moon if 
observed in the part of the sky closer to the sun, so the same object would look fainter at a given distance from Earth impact if observed closer to the sun.) Larger telescopes are needed in the hemisphere facing the sun, but the Air Force GEODDS are adequate to detect them except within 30 degrees of the sun, where twilight (in addition) makes their detection difficult. The GEODDS could be used in a very fast scan mode in the hemisphere away from the sun, since they do not have to go down to as faint a magnitude, and in slower scan mode nearer the sun. Objects that will impact Earth have very little motion across the sky during their final few days before impact. They would best be detected using parallax, the difference in their apparent position on the sky when observed from two different sites on Earth. Such a detection system will require a series of sites around the Earth.

Objects larger than $1 \mathrm{~km}$ in diameter need to be detected months to years before impact to allow time for deflection away from the Earth. Proposed surveys for finding them, such as Spaceguard, will attempt to find all ECAs of this size rather than just those that will hit Earth in the next few years. These surveys plan to concentrate their search towards solar opposition (where the objects look full), as have most surveys to date. This limitation in sky coverage may produce severe selection effects, as is evident in the preliminary analysis of the detection capabilities of the Spaceguard survey. This analysis showed that the survey would find $90 \%$ of all Near-Earth Asteroids (NEAs) larger than $1 \mathrm{~km}$ in diameter after 25 years of searching if undiscovered NEAs have orbits similar to those of known ones. However, the survey would find only about $50 \%$ of the ATENs, which are ECAs with semi-major axes less than 1 astronomical unit (AU). Since existing surveys such as Spacewatch concentrate their search towards opposition, they are likely to suffer the same selection effect, so Atens are underrepresented among known ECAs.

Those ECAs that will hit Earth in the next few years should be easier to detect than other ECAs because they tend to make much closer approaches to Earth in the last few orbital revolutions before impact than does the average ECA. ECAs in nonintersecting orbits are much harder to detect, but they are of no immediate danger to Earth. We find that if we concentrate the search on only those larger objects that could hit Earth in the next 100 years, the problem is much easier than that proposed for Spaceguard, which would find all large ECAs.

We find that the impacting objects do tend to make close approaches to Earth and be much brighter than other ECAs. This is particularly true of ATENs, which have orbital periods less than 1 year, so they can make several close approaches to Earth in the 25 years or so before impact. A telescope search that only goes to magnitude 14 would find all threatening ATENs larger than $1 \mathrm{~km}$ in diameter in a 25-year search if it searched the entire sky farther than 90 degrees from the sun. This magnitude limit is easily attained by telescopes only a few inches in diameter, such as that proposed earlier for the terminal detection of smaller objects. 
inches in diameter, such as that proposed earlier for the terminal detection of smaller objects. The proposed Spaceguard system would go to magnitude 22, but it would cover such a small region centered on the solar opposition point that it would find less than $30 \%$ of these ATENs. About $90 \%$ of all impacting asteroids with semi-major axes greater than $1 \mathrm{AU}$ (Apollos) would also be found by a survey that goes to magnitude 18-20, if it covers the entire sky farther than 90 degrees from the sun. Such a survey could be carried out by the Air Force GEODD telescopes. It would be much more effective than Spaceguard because of the latter's limited sky coverage.

The most difficult objects to detect in sufficient time to allow for their deflection are those larger than $1 \mathrm{~km}$ in diameter with long semi-major axes (long-period asteroids and comets). They cannot make several close approaches to Earth before impact. They could only be detected in the final approach to Earth impact, so they would have to be detected many astronomical units from Earth, where they are quite faint. The Spaceguard magnitude limit of 22 is not adequate to detect them in sufficient time to allow deflection. Because the sky background is about 22 magnitudes/(arc sec) ${ }^{2}$ and atmospheric distortion spreads an asteroid image out to a diameter of at least one second of arc, the only practical way of going to fainter magnitudes is to observe from space, where the diffraction limit of even moderate aperture telescopes is less than $1 \mathrm{sec}$. This suggests an observing triage where the initial all-sky, ground-based surveys would only go down to magnitude 18-20, but would cover the entire sky a few times a week. They would find more than $90 \%$ of the Earth-crossing asteroids in Earth-intersection orbits. It does not make sense to go down to magnitudes fainter than 20 at high marginal cost unless the survey addresses the detection of long-period comets, which requires a system that can go much fainter than magnitude 22 to detect them in sufficient time to allow deflection. A wide-field 2-meter space telescope would go down to magnitude 27 before sky saturation, which would be adequate to detect comets a few years to impact. This would be much more expense than ground-based telescopes, but it would eventually be needed.

\section{Publications}

1. Hills, J. G. and R. Carlson, "Statistics of Large Meteors Detected by Surveillance Satellites," Bull. Am. Astron. Soc., in press (1995).

2. Hills, J. G. and P. J. T. Leonard, "Earth-Crossing Asteroids: The Last Days Before Earth Impact," Astron. J. 109, 401 (1995). 